\title{
EVALUATION OF UNDERGROUND STRUCTURES SUBJECTED TO SEISMIC LOADS
}

\author{
JAN PRUŠKA \\ Czech Technical University in Prague, Faculty of Civil Engineering, Department of Geotechnics, Thákurova 7 , \\ 16629 Prague, Czech Republic \\ correspondence: Pruska@fsv.cvut.cz
}

\begin{abstract}
The paper is focused on the evaluation of the effect of earthquakes on underground structures. Free-field analysis is one solution of this task common mainly in engineering tunnelling practice, but it has some rather simplified aspects (e.g. equivalent shear strain is constant). Pseudostatic finite element calculation combines free-field analysis and the advantages of a FEM model. Dynamic effects are introduced in the form of displacements prescribed along the vertical boundaries of the FEM model in a usually static manner. This approach also implies constant material parameters for the geological profile in the horizontal direction, an arbitrary geometry of excavation, soil structure interaction and description of share waves as a time-dependent 1D analysis of the so called free-field column. Moreover, there is shown an example comparing pseudo-static FEM analysis with an analytical method. Finally, the advantages of the pseudo-static FEM method are presented.
\end{abstract}

KEYwords: Finite element method, earthquake, 1D free field analysis, dynamics, pseudo static analyses.

\section{INTRODUCTION}

The response of underground structures subject to seismic load can be solved in several ways, which can be roughly classified into four categories: laboratory testing, analytical solutions, pseudo static finite element calculations and full dynamic analysis.

(1.) Centrifuge modelling techniques allow well for control testing conditions regarding investigations of soil structure interaction in simulated seismic events. However, there are several difficulties for a dynamic centrifuge model such as accurate modelling of the boundary conditions or the scaling law for dynamic velocity.

(2.) Most simplified methods were derived from closed form solutions but involving neglect of some factors. In general, the methodology of the analytical method is that the seismic loads for an underground structure are characterized in terms of strains imposed on the structure by the surrounding ground or their interaction. Although this is the most conservative method having only a limited application, it does provide a first-order estimate of the liming stresses so to potentially serve as an accuracy check on more complex calculations.

(3.) A pseudo-static finite element calculation provides a solution for any shape of underground structure in a generally non-homogeneous layered rock/soil mass with a potentially nonlinear material response. The basic idea of this approach is that the maximum shear deformation in a free-field condition of the layer represents the maximum dynamic earthquake stress in this layer and can be an input to the numerical model as a boundary condition.
The shear strain equivalent to the actual dynamic loading can be calculated from the particle velocity estimated either in a simplified manner based on limited information or from the solution of a one dimensional simulation of free-field response to an actual earthquake.

(4.) Full dynamic analyses are based on a direct application of recorded accelerations to the actual computational model of the tunnel where the soil and tunnel responses are mechanically coupled and analysed via numerical modelling, such as finite element methods.

\section{AnAlytical Method}

The methodology of the analytical method for a seismic loading design is based on the principle that the static condition has to include the additional loading imposed by seismicity. In the case of underground structures, the seismic loads are described in terms of strains imposed on the structure by the surrounding ground mass or their interaction.

\subsection{Seismic coefficient method}

This method was originally developed for dynamic analysis of over ground buildings. Inertial forces due to earthquakes $F_{1}$ of the structure and $F_{2}$ of the upper soil mass are described by the following equations:

$$
\begin{aligned}
& F_{1}=\frac{a}{g} \cdot Q=K_{c} \cdot Q, \\
& F_{2}=\eta_{c} \cdot K_{h} \cdot m_{1} \cdot g .
\end{aligned}
$$

This method is not suitable for tunnels with large diameters because this method does not reflect the real dynamic property of tunnel structure. 


\subsection{FREE - FIELD DEFORMATION APPROACH}

Tunnel structures are assumed to move together with the surrounding soil/rock mass during earthquakes and therefore undergo the following deformations:

- ovaling,

- axial,

- curvature.

The ovaling deformation of a circular tunnel lining is primarily caused by seismic waves that propagate perpendicular to the longitudinal axis of the tunnel (Figure 1). The vertically propagating shear waves produce usually the most critical ovaling distortion of the lining. The free field deformation approach assumes that the deformation of the underground structure should conform to the deformation of the soil in the free field under seismic shaking. In these simplified methods described in [1, 4 the soil - structure interaction and the effects of the waves are neglected. Moreover the maximum shear strain $\gamma_{\max }$ (Equation 6) induced by shear waves propogating in vertical planes is constant along the depth $z$. This may lead to an overestimate of tunnel lining deformations especially in loose rock and soils.

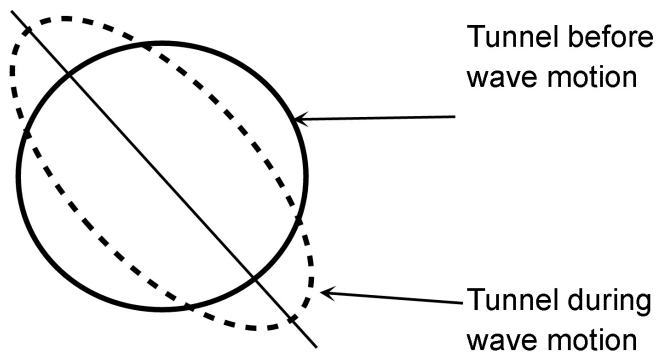

Figure 1. Ovaling deformation of a Circular Cross Section.

In the case of a circular tunnel in the homogeneous medium the ovaling deformation is determined by the following steps:

(1.) according to the Seismic Report we get the Pick Ground Acceleration (3) and the site-specific PickGround-Acceleration (4):

$$
\begin{gathered}
a_{g R}=40 \% \cdot S_{s}, \\
a_{\max , s}=S \cdot a_{g R}
\end{gathered}
$$

(2.) and then we determine the pick acceleration at the depth of the tunnel:

$$
a_{z, \max }=C \cdot S \cdot a_{g R},
$$

(3.) $a_{z, \max } \max$ is used to determine maximum shear deformation in free-field condition as shown in equations (6) and (7):

$$
\begin{gathered}
\gamma_{\max }=\frac{V_{z}}{C_{s}}, \\
V_{z}=k \cdot a_{z, \max }
\end{gathered}
$$

(4.) and finally we obtained the ovaling deformation of the circular tunnel:

$$
\Delta d_{f f}=\gamma_{\text {mean }} \frac{d}{2}
$$

\subsection{SoIL-STRUCTURE INTERACTION APPROACH}

The kinematic interaction between the tunnel and the surrounding soil/rock mass is used to improve the accuracy of the widely used free-field methods. Generally, analytical solutions for estimating soil-structure interaction for tunnels are based on the following assumptions:

- the tunnel is circular;

- the soil is an infinite, elastic, homogeneous and isotropic medium;

- the tunnel lining is generally an elastic tube under plane strain conditions or it is based on the theory of an elastic beam on an elastic foundation;

- the interface between the soil and the tunnel lining has a full-slip or a no-slip condition;

- the soil-structure interaction effects operate in a quasi-static manner, ignoring any inertial interaction effect.

There exist many approaches in the technical literature describing seismic design and analysis of underground structures using a soil structure interaction approach. For example: Billota et al. [5] discussed four different closed-form elastic solutions to evaluate the maximum shear strain $\gamma_{\max }$ (all approaches are based on the equilibrium of the soil column from the surface to the given depth $z$ ), while expressions for displacements and internal lining forces in the circular tunnels excavated in a rock mass with a near fault are presented in [6], and closed-form expressions to calculate the circular tunnel liner forces due to the compressive seismic P-wave propagation are described by Kouretzis et al. [7.

In addition an analytical solution for estimating internal forces in the lining of a circular tunnel in the homogeneous medium is published in [8, 9]. The ovaling deformation is determined by the assumed shear strain $\Delta d$ (Figure 1). We get for parameters $\Delta d_{f f}, \Delta d_{l i n}$ the follow-on formulas:

$$
\begin{gathered}
\Delta d_{\text {lin }}=R^{n} \Delta d_{f f}, \Delta d_{f f}=\gamma_{\text {mean }} \frac{d}{2}, \\
R^{n}=\frac{4\left(1-\nu_{m}\right)}{\alpha^{n}+1}, \alpha^{n}=\frac{12 E_{l}(5-6)}{d^{3} G_{m}\left(1-\nu_{l}^{2}\right)} .
\end{gathered}
$$

It is evident that the material and geometric parameters $R^{n}$ and $\alpha^{n}$ take into account the rigidity of the subsoil-lining system. Expressing the curvature of an elliptical excavation using $\Delta d_{l i n}$ and parameters describing soil structure interaction then the moment in the lining (Figure 2) can be written using the polar 
coordinate $\varphi$ (positive clockwise from horizontal) as:

$$
M(\varphi)=\frac{6 E_{l} \Delta d_{l i n}}{d^{2}\left(1-\nu_{l}^{2}\right)} \cos \left(2\left(\varphi+\frac{\phi}{4}\right)\right) .
$$

With reference to Figure 2 (c), the normal force on the curved element can be expressed as:

$$
N(\varphi)=-\frac{1}{R} \frac{d M^{2}}{d \varphi}+P_{n} R
$$

and after rearranging using equation 10 :

$$
N(\varphi)=\frac{48 E_{l} I \Delta d_{l i n}}{d^{3}\left(1-\nu_{l}^{2}\right)} \cos \left(2\left(\varphi+\frac{\phi}{4}\right)\right)+R p_{n}
$$

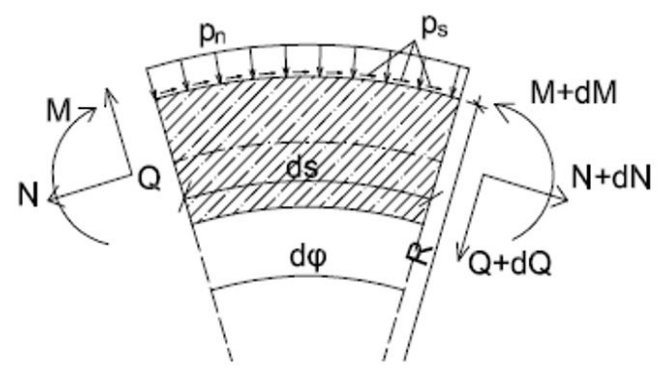

FiguRE 2. Internal forces and contact stress on the differential element.

\section{PSEUDO STATIC FEM}

Implementation of free-field analysis results into the static FEM analyses can be made in several ways. Generally, the shear strains in the 2D solution without lining have to be in conformity with $1 \mathrm{D}$ free-field analyses and the shear deformation in the free-field deformation is applied by prescribed displacements at the boundaries of the numerical model.

In the first method, we consider a homogeneous soil/rock mass with an effective shear modulus G. We determine the maximum shear deformation in free-field condition $\gamma_{\max }$ (6). The value of $\gamma_{\max }$ corresponds to the maximum horizontal displacement applied in the boundaries of the numerical model (Figure 3), calculated by equation:

$$
\Delta x_{\max }=\gamma_{\max } \cdot h_{m} .
$$

The second method considers the layered soil/rock mass (Figure 4) where some sort of homogenization is needed. Adopting the Voigt assumption of constant deformation at each point of the solid we obtained effective shear modulus $G_{V}^{\text {hom }}$ :

$$
G_{V}^{h o m}=\frac{h_{1}}{h} G_{1}+\frac{h_{2}}{h} G_{2}
$$

and average shear deformation of the numerical model $\gamma_{\text {mean }}$ :

$$
\gamma_{\text {mean }}=\frac{h_{1}}{h} \gamma_{1}+\frac{h_{2}}{h} \gamma_{2}
$$

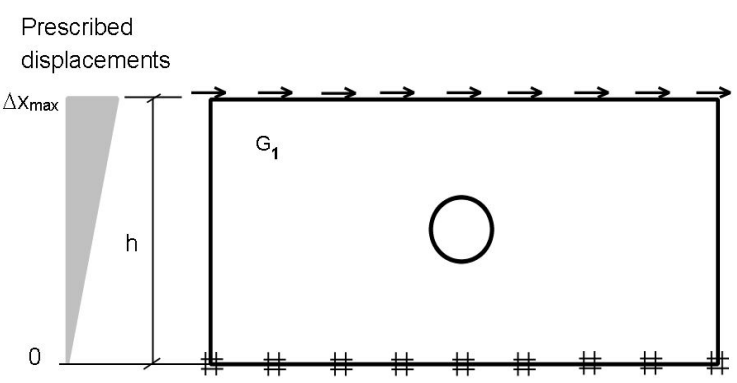

FiguRE 3. Homogenized mass and constant shear strain.

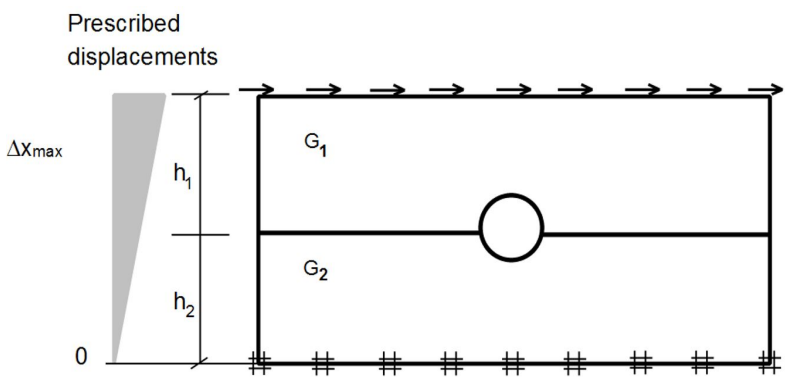

FiguRE 4. Layered mass and constant shear strain .

For the layered mass the better method is based on the setup constant corresponding movements by parts - each layer has a different shear deformation - Figure 5. The prescribed shear deformation values should be in the ratio of the stiffness of the materials in layers to ensure the continuity of the stress at the layer interface:

$$
\frac{\gamma_{1}}{\gamma_{2}} \approx \frac{G_{2}}{G_{1}}
$$

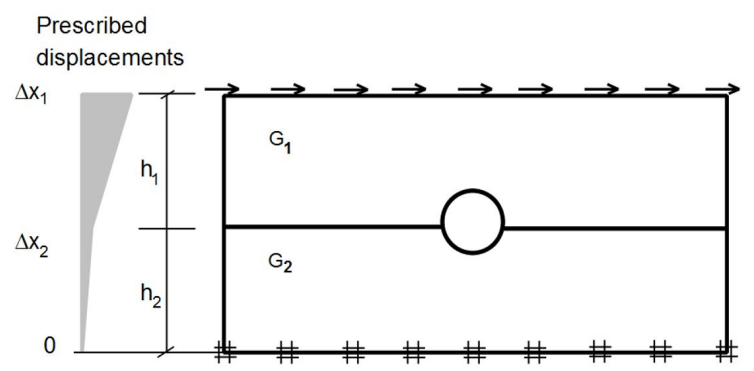

FigURE 5. Layered mass with different values $\gamma$ at layers 1 and 2 .

\section{Full DYNAMiC CALCULATiOnS USING FEM}

The numerical solution of dynamic load effects using the finite element method is based on the principle of virtual displacements, which express the external volume load by the effects of inertial forces and leads to equations of differential motion in the form:

$$
\mathbf{M} \ddot{u}(t)+\mathbf{C} \dot{u}(t)+\mathbf{K} u(t)=F(t) .
$$


The assembly of the stiffness matrix $\mathbf{C}$ is usually a very complicated task thus the Rayleigh formulation is used i.e. as a linear combination of the mass tensor $\mathbf{M}$ and the stiffness tensor $\mathbf{K}$ :

$$
\mathbf{C}=\alpha \mathbf{M}+\beta \mathbf{K} .
$$

Coefficients $\alpha$ and $\beta$ have been calculated according to the double frequency method that avoids an overestimate of damping throughout the considered frequency range [10]. Assuming that the first natural frequency is the least damping, the equation (17) can be decomposed to the natural vibrations. Using the damping ratio $\xi$ we get a relationship for individual frequencies (for real systems with a large number of degrees of freedom):

$$
\xi_{i}=\frac{1}{2}\left(\frac{\alpha}{\omega_{i}}+\beta \omega_{i}\right) .
$$

In order for there to be a damping ratio $\xi$ minimal for $\omega=\omega_{i}$ it must fulfill the next equation:

$$
\frac{d \xi}{d \omega}=\frac{1}{2}\left(-\frac{\alpha}{\omega_{i}}+\beta\right)
$$

After substitution $\omega=\omega_{i}$ into equation 20 wet

$$
\alpha=\omega_{1}^{2} \beta
$$

and then from equation 20

$$
\beta=\frac{\xi_{1}}{\omega_{1}} .
$$

In common practice, equation 17 is solved by direct integration using the Newmark integration method. The discrete form of equation (17) already includes the influence of boundary conditions and the effect of loading in the form of a prescribed acceleration of displacements at the lower limit.

\section{CASE eXemplification}

The FEM calculation results will be compared with the results of the analytical calculation published in [11. The geometry of the model and the finite element mesh is shown in Figure 6 and Table 1 contains the parameters of the subsoil and the tunnel lining. The seismic loading has PGA $2 \mathrm{~ms}^{-2}$ and the wave period is $0.01 \mathrm{~s}$. All FEM calculations were made by Geo5 Tunnel software [12]. For the sake of clarity the representative extreme values obtained for $\varphi=\frac{\phi}{4}$ have been set - see Table 2. For illustration the resulting moment in the lining is also shown in Figure 7

The two approaches in terms of the absolute percentage of the difference in the last column of Table 2 can be mutually assessed. It is evident that in the case of a homogeneous mass and a circular excavation both methods give comparable results. The exception is the value of normal force in the case without a considering of the interaction between the lining and the soil (effect of the contact stress). If we take into account the value of contact stress in the equation (18), the resulting values $\Delta d_{f f}$ a $\Delta d_{l i n}$ of normal force for both methods are almost identical.

\begin{tabular}{llll}
\hline Type & Unit & Value & Unit \\
\hline Loading & $\gamma_{\text {mean }}$ & 0.0105 & {$[-]$} \\
\hline Soil & $G_{m}=G^{\text {hom }}$ & 8.65 & {$[\mathrm{MPa}]$} \\
\hline & $\nu_{m}$ & 0.3 & {$[\mathrm{~Pa}]$} \\
\hline & $\rho_{m}$ & 2000 & {$\left[\mathrm{~kg} / \mathrm{m}^{3}\right]$} \\
\hline Lining & $\gamma_{\text {mean }}$ & 0.0105 & {$[-]$} \\
\hline & $\nu_{l}$ & 0.3 & {$[-]$} \\
\hline & $G_{l}$ & 12.5 & {$[\mathrm{GPa}]$} \\
\hline $\mathrm{d}$ & 6 & {$[\mathrm{~m}]$} \\
\hline $\mathrm{h}$ & 0.3 & {$[\mathrm{~m}]$} \\
\hline $\mathrm{I}$ & 0.00225 & {$\left[\mathrm{~m}^{4}\right]$} \\
\hline
\end{tabular}

TABLE 1. Input parameters of the numerical model

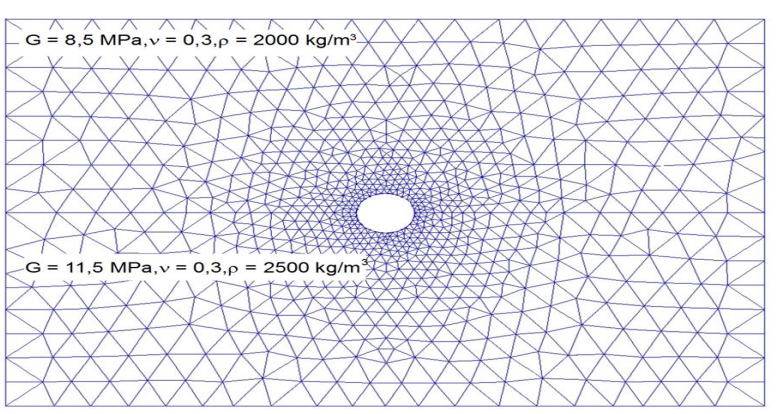

Figure 6. Numerical model.

\section{Conclusions}

The paper is focused on the evaluation of the seismic effects on the tunnel lining by free-field analysis and pseudo static calculation using FEM. Generally, both calculation methods give comparable results in the case of a homogeneous soil environment and ignoring soil structure interaction. However, from a practical point of view, the FEM combined with the 1D freefield analysis yields clear advantages:

- It allows a possibility to derive the load for any time of the earthquake (measured or synthetically generated accelerograms).

- It analyses any shape of excavation (lining).

- It takes into account the potentially non-linear subsoil response.

- It take into consideration the layered rock/soil mass depending on the "real" material parameters.

Another indisputable advantage is taking into account the soil structure interaction whose neglect in the case of an analytical method may lead to a significant overestimation of the normal force. Such a non-realistic value of the normal force leads to a lower degree of reinforcement in the effects of the bending moment for the considered cross section. 


\begin{tabular}{llll}
\hline Variable & FEM & Analytical & Difference [\%] \\
\hline$\Delta d_{f f}[\mathrm{~mm}]$ & 31.3 & 31.5 & 0.6 \\
\hline$\Delta d_{\text {lin }}[\mathrm{mm}]$ & 31.0 & 36.1 & 14.1 \\
\hline $\mathrm{M}[\mathrm{kNm} / \mathrm{m}]$ & -367.6 & -422.8 & 13.1 \\
\hline $\mathrm{V}[\mathrm{kN} / \mathrm{m}]$ & 244.5 & 281.0 & 13.0 \\
\hline $\mathrm{N}[\mathrm{kN} / \mathrm{m}]$ & -380.8 & -562.0 & 32.2 \\
\hline $\mathrm{N}^{*}[\mathrm{kN} / \mathrm{m}]$ & -380.8 & $-390.1^{*}$ & $2.4^{*}$ \\
\hline$*$ The influence of cont. & nor. stresses was taken into the calc.
\end{tabular}

TABLE 2. Output parameters.

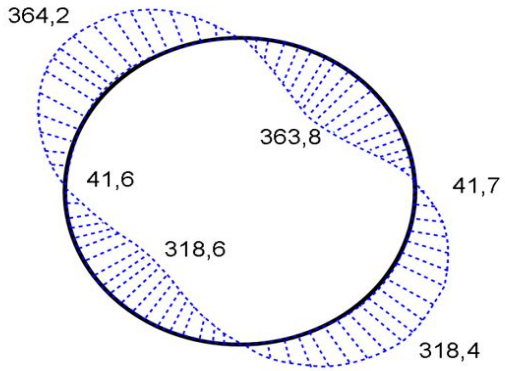

Figure 7 . Moment in the lining.

\section{LIST OF SYMBOLS}

$a$ earthquake acceleration

$a_{n}$ ratio between the stiffness of lining and massive

$a_{z, \max }$ pick acceleration at the depth of the tunnel

$d$ diameter of the excavation

$g$ gravitational acceleration

$h$ thickness of the lining

$k$ ratio of $\mathrm{PGV}$ to $\mathrm{PGA}$

$m_{1}$ weight of soil mass

$u$ actual displacement vector

$C$ ground motion at depth z

C damping matrices

$C_{s}$ apparent propagation velocity of S-wave

$D$ tunnel radius

$E_{l} \quad$ Young modulus of the lining

$F \quad$ vector of nodal loading

$G$ shear modulus of soil/rock

$G_{m}$ elasticity modulus of the massive

$I$ moment of inertia of the lining

K stiffness matrices

$K_{v}$ seismic coefficient in vertical direction

$K_{h}$ seismic coefficient in horizontal direction

M mass matrices

$Q \quad$ weight of structure

$P G A$ peak ground acceleration

$R$ reduction factor of depth influence

$R$ radius of excavation

$R_{n}$ lining - massive racking ratio under normal loading

$S$ coefficient based on the types of elastic response spectra suggested in Eurocode 8
$S_{s}$ short period spectral acceleration

$V_{s}$ peak ground velocity

$\alpha$ parameter that determines the influence of mass in the damping of systems

$\alpha \mathbf{M}$ damping proportional to displacements

$\beta$ parameter that determines the influence of the stiffness in the damping of the system

$\beta \mathbf{K}$ damping proportional strain rate

$\gamma_{\text {mean }}$ average shear strain of the model

$\eta_{c}$ integrate response coefficient related to the tunnel depth, soil property and project significance

$\nu_{m}$ Poisson number of the massive

$\nu_{l} \quad$ Poisson number of the lining

$\sigma_{v}$ Poisson number of the lining

$\omega$ angular frequency

$\omega_{1}$ angular frequency of the first natural mode

$\omega_{i}$ angular frequency of i-th mode

\section{ACKNowledgements}

The article was produced with financial support from the Competence Centres Program of the Technology Agency of the Czech Republic (TA CR), project no. TE01020168 Centre for Effective and Sustainable Transport Infrastructure (CESTI).

\section{REFERENCES}

[1] J.-N. Wang. Seismic design of tunnel - A simple state of the art design approach. Parsons, Brinckerhoff, Quade \& Douglas, Inc. 1993. Monograph 7.

[2] M. S. Power, D. Rosidi, J. Kaneshiro. Strawman: screening, evaluation, and retrofit design of tunnels. National Center for Earthquake Engineering Research, Buffalo, New York. Report Draft. Vol. 3.

[3] J. Penzien, C. Wu. Stresses in linings of bored tunnels. International Journal of Earth-quake Engineering and Structural Dynamics 3(27):283-300, 1998.

[4] Y. M. A. Hashash, J. J. Hook, B. Schmidt, J. I.-C. Yao. Seismic design and analysis of under-ground structures. Tunnelling and Underground Space Technology (16):247-293, 2001.

[5] E. Bilotta, G. Lanzano, G. Russo, et al. Pseudo-static and dynamic analyses of tunnels in transversal and longitudinal directions. In Proceedings of 4 th International Conference on Earthquake Geotechnical Engineering. ICEGE, Thessaloniki, 2007. 
[6] M. Corigliano, L. Scandella, C. Lai, R. Paolucci. Seismic analysis of deep tunnels in near fault conditions: a case study in southern italy. Bulletin of Earthquake Engineering 9(4):975-995, 2011.

[7] G. Kouretzis, K. Andrianopoulos, S. Sloan, J. Carter. Analysis of circular tunnels due to seismic p-wave propagation, with emphasis on unreinforced concrete liners. Computers and Geotechnics 55:187-194, 2014.

[8] AASHTO. Technical manual for design and construction of road tunnels - civil elements. First Edition. American Association of State Highway Transportation Officials, 2010.
[9] C. Hunk, J. Monses, N. Munfah, J. Wisniewski. Technical manual for design and construction of road tunnels - civil elements. First Edition. Federal Highway Administration, 2009. Report FHWA-NHI-10-034.

[10] G. Lanzo, A. Pagliaroli, B. D'Elia. Influenza della modellazione di rayleigh dello smorzamento viscoso nelle analisi di risposta sismica locale. In Proceedings of 11th National Conference L'ingegneria Sismica in Italia. 2004.

[11] D. Kučera. Posouzení geotechnické konstrukce na seismické zatížení. Diplomová práce. ČVUT v Praze, Fakulta stavební, 2017. In Czech.

[12] GEO 5 FEM. Online, available from: https: //www.fine.cz/geotechnicky-software/mkp-tunel/ 